\title{
Effects of Normal Morphology Sperm Count on Fertilization Time in Infertile Couples with Teratospermic Males: An Evaluation with an Embryoscope
}

\author{
(1) Elif Ganime Aygun1, (1) Emine Karabük2 \\ ${ }^{1}$ Acıbadem Mehmet Ali Aydınlar University, Atakent Hospital, Clinic of Obstetrics and Gynecology, Istanbul, Turkiye \\ ${ }^{2}$ Acıbadem Mehmet Ali Aydınlar University, Faculty of Medicine, Department of Obstetrics and Gynecology, Istanbul, Turkiye
}

\section{What's known on the subject? and What does the study add?}

Embryo development depends on both egg and sperm quality. This study examines the effects of sperm morphology on embryo development. Emphasizes that factors other than the visual evaluation in sperm are effective in embryo development.

\begin{abstract}
Objective: Incubation of the embryo is the vital step in assisted reproductive techniques. Embryoscope systems are incubators that keep the embryo stable and allow for continuous observation without opening the lid. Therefore, the embryo is not displaced and goes through a temperature or air change. The primary aim of this study was to explore the effects of normal morphology sperm count on two pronucleus formation time following intracytoplasmic sperm injection in infertile couples with teratospermic males. The secondary aim was clinical pregnancy.

Materials and Methods: Sixty-seven couples undergoing in vitro fertilization were included in this retrospective cohort study. First, intracytoplasmic sperm injection was performed to metaphase II-oocytes. Then, the embryos were placed in an embryoscope (a time-lapse system) for observation. The time that embryos demonstrated two pronuclei were recorded. Demographic and reproductive data were obtained and analyzed.

Results: A statistically significant weak correlation was detected between sperm morphology the two pronucleus formation times ( $r=0.295$, $p=0.017$ ). Severely decreased normal sperm count was significantly associated with pregnancy with less decreased normal sperm count ( $p>0.024)$. Moreover, decreased two pronucleus formation time was significantly associated with pregnancy.

Conclusion: A statistically significant, albeit weak, correlation was detected between sperm morphology with two pronucleus formation time. Furthermore, having a lower normal sperm count was related to a higher pregnancy rate than having a higher normal sperm count. Lastly, reduced two pronucleus formation time was significantly associated with pregnancy.
\end{abstract}

Keywords: Embryoscopes, fertilization, infertility, in vitro fertilization, pregnancy, sperm, time-lapse imaging

\section{Introduction}

There has been a breakthrough in assisted reproductive techniques with the discovery of in vitro fertilization in 1978. This discovery has enabled approximately three to four times assisted pregnancies than spontaneous pregnancies. As a result, multiple embryo transfers were carried out to increase this number even more. However, this has led to various complications for the mother and the baby, such as ectopic and multiple pregnancies $(1,2)$. Subsequently, physicians started practicing single embryo transfers. Therefore, a need for improving embryo selection was born, and technological advances pursued this need appropriately.

Current studies demonstrate the impact of the sperm on the embryo quality in addition to the oocyte $(3,4)$. Evaluation of the movement, DNA fragmentation, morphological abnormalities of the sperm are determining factors in proper fertilization, implantation, embryo development, and subsequently,

Correspondence: Elif Ganime Aygun MD, Acıbadem Mehmet Ali Aydınlar University, Atakent Hospital, Clinic of Obstetrics and Gynecology, İstanbul, Turkiye E-mail: elif.aygun@acibadem.com ORCID-ID: orcid.org/0000-0003-3737-7250

Received: 03.01.2022 Accepted: 14.01 .2022

Cite this article as: Aygun EG, Karabük E. Effects of Normal Morphology Sperm Count on Fertilization Time in Infertile Couples with Teratospermic Males: An Evaluation with an Embryoscope. J Urol Surg, 2022;9(2):76-81.

๑Copyright 2022 by the Association of Urological Surgery / Journal of Urological Surgery published by Galenos Publishing House. 
pregnancy (5-7). Therefore, the morphology of the sperm is crucial when it comes to focusing on embryo development. Additionally, studies have demonstrated the relationship between sperm quality and embryo development (8). Especially, abnormalities in the morphology of the sperm could cause fertilization defects and low cleavage-stage problems (9).

Incubation of the embryo is the vital step in assisted reproductive techniques. Furthermore, no evidence of conventional techniques being superior to embryoscope has able be shown by systematic reviews and meta-analysis $(10,11)$. Embryoscope systems are incubator systems that keep the embryo stable and allow for the continuous observation of embryo development without the need to open the lid. Therefore, the embryo is not displaced and go through a heat change. It is one of the most significant advantages of embryoscopes over conventional methods $(12,13)$. Furthermore, the time-lapse imaging technology that enables continuous recording utilized by embryoscopes could shed light on the relationship between sperm parameters and embryo development and extend the currently limited literature (14).

The primary aim of this study was to explore the effects of normal morphology sperm count on two pronucleus formation time following intracytoplasmic sperm injection in infertile couples with teratospermic males. The secondary aim of the study was to achieve clinical pregnancy.

\section{Materials and Methods}

This study was authorized by the Acibadem Mehmet Ali Aydinlar University Research Ethics Committee (ATADEK) with the decision number: 2021-21/06 on 04/11/2021. The study was conducted at Acibadem Mehmet Ali Aydinlar University Atakent Hospital, Department of Obstetrics and Gynecology, Istanbul (Turkey), between 2015 and 2016. All methods were performed following the relevant guidelines and regulations.

\section{Study Design}

A research question and hypothesis were developed:

Research Question: In a population of infertile couples with teratospermic males, is normal morphology sperm count associated with two pronucleus formation time following intracytoplasmic sperm injection?

Study Hypothesis: In a population of infertile couples with teratospermic males, increased normal morphology sperm count is associated with decreased two pronucleus formation time following intracytoplasmic sperm injection

The study was designed as a retrospective cohort study. Age, karyotypic analysis, sperm morphology, number of oocytes, two pronucleus formation time, the status of pregnancy, and the genetic testing status of the parents were obtained and analyzed. The data were collected from the hospital's electronic health record system.

The primary aim of this study was to explore the effects of normal morphology sperm count on two pronucleus formation time following intracytoplasmic sperm injection in infertile couples with teratospermic males. The secondary aim of the study was to achieve clinical pregnancy.

\section{Selection and Description of Participants}

The inclusion criterion for couples was infertility (failure to conceive after one year of unprotected coitus) were

- For males:

o Having $>10 \%$ motile sperm in semen

o Having $\leq 4 \%$ normal morphology sperm

o Being older than 18 years old

- For females

o Being 18-43 years old The exclusion criteria were

- For males:

o Azoospermia (zero sperm present under light microscopy evaluation)

o Any genetic defects (determined by karyotypic analysis)

- For females:

o Being older than 43 years old (due to extremely low chances of fertilisation success)

o Severe ovarian failure (zero antral follicle count on transvaginal ultrasonography and FSH $>\times 15 \mathrm{IU} / \mathrm{L}$ and $\mathrm{LH}>10$ $\mathrm{IU} / \mathrm{L}$ and/or amenorrhea) (due to extremely low chances of fertilisation success)

o Severe endometriosis (detected by vaginal examination and transvaginal ultrasonography or diagnostic laparoscopy) (due to extremely low chances of fertilisation success)

o Any genetic defects (determined by karyotypic analysis)

\section{Sperm Evaluation}

Semen samples were collected from males by masturbation after three days of sexual abstinence. First, the samples were treated with $\mathrm{ORIGIO}^{\circledR}$ Sperm Wash (reference number: 84050060A; Måløv, Denmark) with a 1:1 ratio in Falcon conical tubes and centrifuged at 1500 revolutions per minute for $10 \mathrm{~min}$ at 37 ${ }^{\circ} \mathrm{C}$ to concentrate the sperm. Afterwards, the fluid on the top is removed using a micropipette. Finally, the sperm was left and evaluated using a light microscope. The scoring of normal morphological sperm to all sperm was made between 1 and 4\%.

\section{Oocyte Collection}

Ovulation was induced in females with 225-450 IU of gonadotropin antagonists [recombinant follicle-stimulating hormone ( $\mathrm{rFSH}$ ) or $\mathrm{rFSH}$ and recombinant luteinising hormone 
$(\mathrm{rLH})$, depending on the number of antral follicles chosen patient by patient basis]. When follicles were measured to 18 $\mathrm{mm}$ by transvaginal ultrasound, recombinant human chorionic gonadotropin alpha (rHCG- $\alpha$ ) (Ovitrelle 250 microgram/0.5 millilitres) was administered subcutaneously to facilitate oocyte expulsion. Afterwards, under general anesthesia, transvaginal ultrasound-guided oocyte pickup was performed. Then, the metaphase II-oocytes were determined under a light microscope, and were placed in Petri dishes containing a single-step medium (CooperSurgical, Inc; Connecticut, United States of America).

\section{Fertilization}

Intracytoplasmic sperm injection was performed to metaphase II-oocytes using a micromanipulation microscope system (Leica Microsystems, Wetzlar, Germany). Afterwards, the embryos were transferred to 20 microliter droplets single step mediums, and lastly, mineral oil was added. Then, the embryos were placed in an EmbryoScope+ time-lapse system (Vitrolife, Gothenburg, Sweden) for observation. The time that embryos demonstrated two pronuclei were recorded. The embryos are followed up until the blastocyst stage (E5), and then they are either implanted, frozen, or underwent trophectoderm biopsy.

\section{Statistical Analysis}

Descriptive statistics are presented using mean, standard deviation, median (and minimum-maximum) for continuous variables. Additionally, frequencies $(\mathrm{N})$ and percentages $(\%)$ are used to represent categorical variables.

Non-parametric statistical methods were used for values with skewed distribution. For comparison of two non-normally distributed groups, the Mann-Whitney U test was used.

Spearman's rho correlation analysis is used to investigate the relationship between two non-normally distributed continuous variables.

Statistical significance is accepted when the two-sided p-value is lower than 0.05 . Statistical analysis was performed using the STATA/SE 17.0 software.

\section{Results}

Sixty-seven patients were included. A single metaphase IIoocyte was selected from the females of the couples $(n=67)$, and ICSI was applied. Table 1 presents the characteristics of the study population.

Primary Outcome (Correlation between normal morphology sperm and two pronucleus formation time)

Table 2 presents the correlation analysis between two pronucleus formation times and normal morphology sperm. The Spearman's rho correlation is interpreted as when the " $r$ " correlation coefficient gets close to 1 ; the correlation gets stronger. The $r$-values between $0-0.3$ are accepted as a weak correlation.

A statistically significant weak correlation was detected between sperm morphology with two pronucleus formation times $(p=0.017)$ (Table 2 and Figure 1$)$.

\section{Secondary Outcome (Pregnancy)}

Table 3 compares pregnancy groups according to normal morphology sperm and two pronucleus formation time.

More decreased normal sperm count was significantly associated pregnancy than less decreased normal sperm count ( $p>0.024)$. Figure 2 and Figure 3 display the boxplots for pregnancy, normal morphology sperm count, and two pronucleus formation time. Moreover, decreased two pronucleus formation time was significantly associated with pregnancy. However, subgroup analysis of sperm morphology and fertilization time did not detect statistical significance $(p=0.067)$ (Table 4$)$.

\section{Discussion}

The primary goal of this study was to investigate whether normal morphology sperm count affected the time it took for two pronuclei to develop after intracytoplasmic sperm injection in infertile couples with teratospermic males. Normal sperm morphology and two pronucleus formation times were found to have a statistically significant weak correlation. Additionally, the study's secondary goal was to achieve clinical pregnancy. Paradoxically, having a lower normal sperm count was related

\begin{tabular}{|c|c|c|c|}
\hline Parameters & n (\%) & Mean + SD & $\begin{array}{l}\text { Median } \\
\text { (min-max) }\end{array}$ \\
\hline Age (Female) & $67(100)$ & $34.2 \pm 4.1$ & $35(23-41)$ \\
\hline $\begin{array}{l}\text { Normal morphology } \\
\text { sperm count }(\%)\end{array}$ & 66 (98.5) & $2.02 \pm 0.92$ & $2(1-4)$ \\
\hline Number of oocytes & $67(100)$ & $11.9 \pm 9.3$ & $10(0-40)$ \\
\hline $\begin{array}{l}\text { Two pronucleus } \\
\text { formation time (hour) }\end{array}$ & $66(98.5)$ & $17.2 \pm 0.9$ & $17(16-19)$ \\
\hline Pregnancy Status & $67(100)$ & & \\
\hline Pregnancy & $45(67.2)$ & & \\
\hline No pregnancy & $20(29.8)$ & & \\
\hline Anembryonic pregnancy & $2(3)$ & & \\
\hline
\end{tabular}

Table 2. Correlation of two pronucleus formation time with age and normal morphologysperm

\begin{tabular}{|l|l|l|}
\hline \multicolumn{2}{|l|}{ Correlation } & $\begin{array}{l}\text { Two pronucleus } \\
\text { formation time }\end{array}$ \\
\hline \multirow{2}{*}{ Normal morphology sperm } & $\mathbf{r}$ & 0.295 \\
\cline { 2 - 3 } & $\mathbf{p}$ & $\mathbf{0 . 0 1 7}$ \\
\hline (r: correlation coefficient) Spearman's rho correlation
\end{tabular}




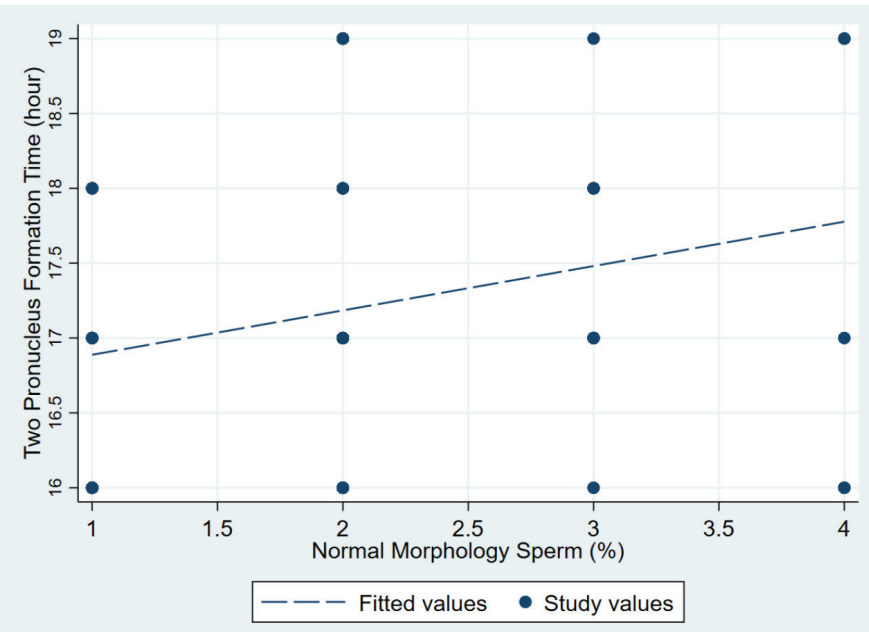

Figure 1. Scatterplot displaying the relationship between normal morphology sperm count and two pronucleus formation time

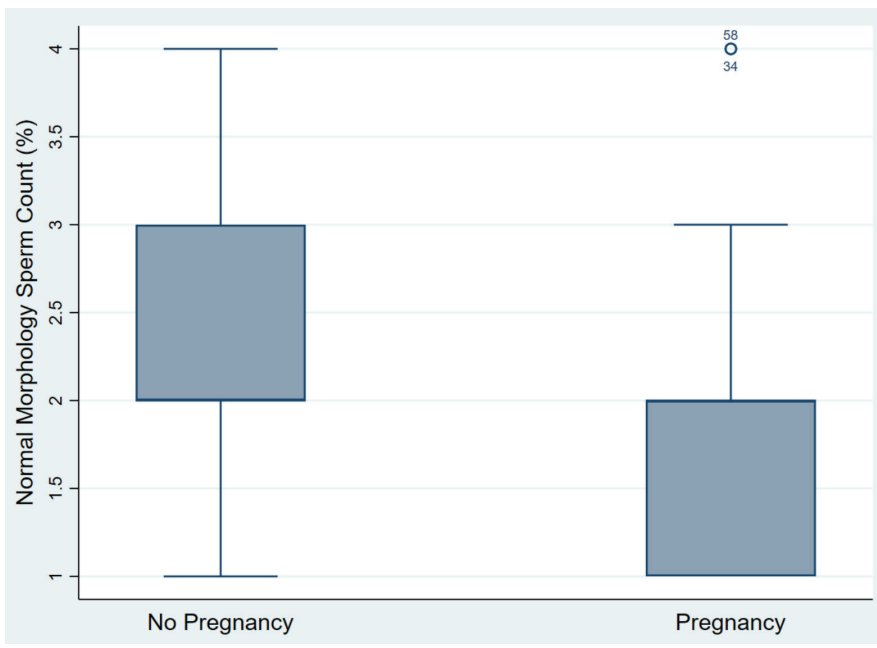

Figure 2. Boxplot of the normal morphology sperm count and pregnancy

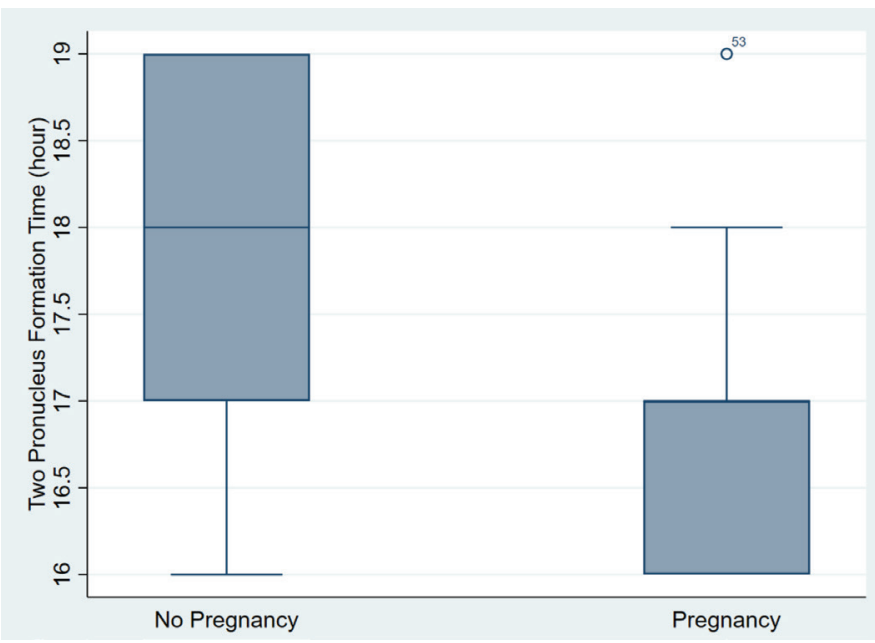

Figure 3. Boxplot of the two pronucleus formation time and pregnancy to a higher pregnancy rate than having a higher normal sperm count.

Many sperm-related factors, including morphology, are known, and more are being investigated to affect many stages of pregnancy, including fertilization, early cleavage of the embryo, and the foetus's formation. It highlights that the oocyte is not the only factor in fertilization and embryo development, and the sperm holds similar significance (15). Abnormal sperm morphology directly affects fertilization failure and pregnancy $(8,16,17)$. Additionally, it is recorded as an essential factor in spontaneous miscarriages (18). A novel study by Nikovola et al. (19) recently investigated morphology among many sperm parameters and concluded that morphological abnormalities of the sperm were closely associated with embryo morphokinetics. Concurrent with this, the results suggest that normal sperm morphology and two pronucleus formation time, albeit weak, have a statistically significant correlation (Table 2).

Previous studies demonstrated a positive correlation between earlier fertilization (two pronucleus formation time) and normal sperm morphology. Moreover, normal sperm was detected to provide adequate implantation and clinical pregnancy results in couples undergoing ICSI $(20,21)$. In contrast, the current study demonstrated that more decreased normal sperm count was significantly associated with decreased two pronucleus formation time and pregnancy than less decreased normal sperm count (Table 3). In another study, no statistically significant difference was detected in clinical pregnancy, embryo implantation, and miscarriage rates between poor morphology and normal morphology sperm (22). The selection of the sperm is a semiquantitative technique entirely at the discretion of the embryologist. Finding quality sperm for ICSI in in vitro fertilization procedures is challenging as the expression "finding a needle in a haystack" goes. Therefore, maximum effort is given to select the best quality sperm among the poor morphologic sperm. In this study, it could be attributed to the fact that a limited number of patients with higher normal morphology sperm count was present (Table 4) and could be considered a limitation in this regard. However, selecting the best sperm in the pool of bad morphology sperm could positively impact the pregnancy rate. The current study's authors' ongoing works on incorporating artificial intelligence into sperm selection could transform this process into a fully quantitative technique.

Time-lapse imaging technology has emerged recently in embryo selection in the form of embryoscopes. It, expectedly, increases clinical pregnancy and live birth rates $(23,24)$. Embryoscopes are incubator systems that allow for the continuous observation of embryo development and keep the embryo stable without opening the lid. Therefore, the embryo is not displaced and protected from trauma and air content, and the temperature inside the incubator does not change. These are the most 


\begin{tabular}{|c|c|c|c|c|c|}
\hline \multirow[t]{2}{*}{ Pregnancy } & \multicolumn{2}{|l|}{ No $(n=20)$} & \multicolumn{2}{|l|}{ Yes $(n=45)$} & \multirow[b]{2}{*}{$\mathbf{p}$} \\
\hline & Mean + SD & $\begin{array}{l}\text { Median } \\
\text { (min-max) }\end{array}$ & Mean + SD & $\begin{array}{l}\text { Median } \\
\text { (min-max) }\end{array}$ & \\
\hline Normal morphology sperm count (\%) & $2.42 \pm 0.96$ & $2(1-4)$ & $1.84 \pm 0.85$ & $2(1-4)$ & 0.024 \\
\hline Two pronucleus formation time (hour) & $17.84 \pm 1.17$ & $18(16-19)$ & $16.89 \pm 0.77$ & $17(16-19)$ & 0.001 \\
\hline
\end{tabular}

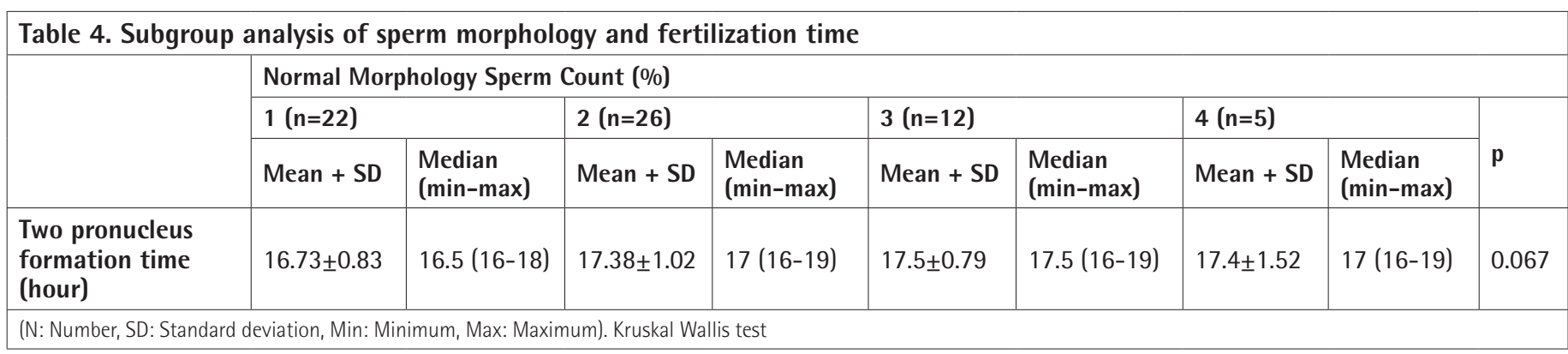

significant advantages of embryoscopes $(12,13)$. The current study utilized embryoscopes and detected a significant association between pregnancy and two pronucleus formation time. Moreover, prolonged fertilization time was determined to have negative effects on pregnancy. Parallel to the current results, recent studies suggested that the delay in two pronucleus formations could cause implantation failures (19).

The current study holds an essential place in investigating the sperm-related issues in in vitro fertilization. The cytoplasm of the sperm and oocyte's and the interactions between the two play a crucial role in embryo development. Moreover, conducting subabnormality analyses, screening DNA fragmentation defects, and even analysing histone and protamine of the sperm might yield positive and decisive results regarding pregnancy. To summarize, sperm-related factors have vital importance in assistedreproductive techniques and require further investigation.

\section{Study Limitations}

The first limitation of this study was its limited sample size. Secondly, the statistical analyses were made based on morphological observations. Moreover, the utilization of ICSI enabled the best sperm to be selected under a light microscope and injected into the oocyte. Thus, it could alone have a positive effect on embryo development in males with teratospermia. Lastly, quantitative analyses such as sub-abnormality analyses, screening for DNA fragmentation defects \& histone and protamine were not performed.

\section{Conclusion}

In conclusion, a statistically significant weak correlation was detected between sperm morphology with two pronucleus formation times (primary outcome). Furthermore, having a lower normal sperm count was related to a higher pregnancy rate than having a higher normal sperm count. Lastly, decreased two pronucleus formation time was significantly associated with pregnancy. However, subgroup analysis of sperm morphology and fertilization time did not detect statistical significance. Therefore, further studies with larger sample sizes investigating additional sperm parameters in infertile couples must be conducted to fully explore the roots of sperm-related issues.

Acknowledgements: The authors want to thank the embryologists Sinem Ercan Doğan, PhD (Director of IVF Laboratory at Acibadem Mehmet Ali Aydinlar University Atakent Hospital) and Gözde Babikli (IVF Laboratory at Acibadem Mehmet Ali Aydinlar University Atakent Hospital) for conducting fertilization procedures. We would also like to thank Erol Barbur, MD, for his professional assistance with the manuscript translation, critical revising, data visualization, and submission processes.

\section{Ethics}

Ethics Committee Approval: This study was authorized by the Acibadem Mehmet Ali Aydinlar University Research Ethics Committee (ATADEK) with the decision number: 2021-21/06 on 04/11/2021.

Informed Consent: Retrospective study.

Peer-review: Externally peer-reviewed.

\section{Authorship Contributions}

Surgical and Medical Practices: E.G.A., Concept: E.G.A., Design: E.G.A., Data Collection or Processing: E.K., E.G.A., Analysis or Interpretation: E.K., Literature Search: B.H., Writing: E.G.A., E.K. 
Conflict of Interest: No conflict of interest was declared by the authors.

Financial Disclosure: The authors declare that they have no relevant financial.

\section{References}

1. Ombelet W, De Sutter P, Van der Elst J, Martens G. Multiple gestation and infertility treatment: registration, reflection and reaction--the Belgian project. Hum Reprod Update 2005;11:3-14.

2. Walker MC, Murphy KE, Pan S, Yang 0 , Wen SW. Adverse maternal outcomes in multifetal pregnancies. BJOG 2004;111:1294-1296.

3. Demko ZP, Simon AL, McCoy RC, Petrov DA, Rabinowitz M. Effects of maternal age on euploidy rates in a large cohort of embryos analyzed with 24-chromosome single-nucleotide polymorphism-based preimplantation genetic screening. Fertil Steril 2016;105:1307-1313.

4. Parinaud J, Mieusset R, Vieitez G, Labal B, Richoilley G. Influence of sperm parameters on embryo quality. Fertil Steril 1993;60:888-892.

5. Lundin K, Söderlund B, Hamberger L. The relationship between sperm morphology and rates of fertilization, pregnancy and spontaneous abortion in an in-vitro fertilization/intracytoplasmic sperm injection programme. Hum Reprod 1997;12:2676-2681.

6. Kruger TF, Coetzee K. The role of sperm morphology in assisted reproduction. Hum Reprod Update 1999;5:172-178.

7. Van Waart J, Kruger TF, Lombard CJ, Ombelet W. Predictive value of normal sperm morphology in intrauterine insemination (IUI): a structured literature review. Hum Reprod Update 2001;7:495-500.

8. Lammers J, Reignier A, Splingart C, Catteau A, David L, Barriere P, Freour T. Does sperm origin affect embryo morphokinetic parameters? J Assist Reprod Genet 2015;32:1325-1332.

9. Janny L, Menezo YJ. Evidence for a strong paternal effect on human preimplantation embryo development and blastocyst formation. Mol Reprod Dev 1994;38:36-42.

10. Chen M, Wei S, Hu J, Yuan J, Liu F. Does time-lapse imaging have favorable results for embryo incubation and selection compared with conventional methods in clinical in vitro fertilization? A meta-analysis and systematic review of randomized controlled trials. PLoS One 2017;12:e0178720.

11. Armstrong S, Bhide P, Jordan V, Pacey A, Marjoribanks J, Farquhar C. Time-lapse systems for embryo incubation and assessment in assisted reproduction. Cochrane Database Syst Rev 2019;5:CD011320.

12. Bhide $P$, Maheshwari $A$, Cutting $R$, Seenan $S$, Patel $A$, Khan $K$, Homburg R. Time lapse imaging: is it time to incorporate this technology into routine clinical practice? Hum Fertil (Camb) 2017;20:74-79.
13. Milewski $R$, Ajduk A. Time-lapse imaging of cleavage divisions in embryo quality assessment. Reproduction 2017;154:R37-R53.

14. Basile N, Meseguer M. Time-lapse technology evaluation of embryo quality and new markers for embryo selection. Expert Review of Obstetrics \& Gynecology 2012;7:175-190.

15. Colaco S, Sakkas D. Paternal factors contributing to embryo quality. J Assist Reprod Genet 2018;35:1953-1968.

16. Zinaman MJ, Brown CC, Selevan SG, Clegg ED. Semen quality and human fertility: a prospective study with healthy couples. J Androl 2000;21:145153.

17. Slama $R$, Eustache $F$, Ducot $B$, Jensen $T K$, Jørgensen $N$, Horte $A$, Irvine $S$, Suominen J, Andersen AG, Auger J, Vierula M, Toppari J, Andersen AN, Keiding N, Skakkebaek NE, Spira A, Jouannet P. Time to pregnancy and semen parameters: a cross-sectional study among fertile couples from four European cities. Hum Reprod 2002;17:503-515.

18. Cao X, Cui Y, Zhang X, Lou J, Zhou J, Wei R. The correlation of sperm morphology with unexplained recurrent spontaneous abortion: A systematic review and meta-analysis. Oncotarget 2017;8:55646-55656.

19. Nikolova S, Parvanov D, Georgieva V, Ivanova I, Ganeva R, Stamenov G. Impact of sperm characteristics on time-lapse embryo morphokinetic parameters and clinical outcome of conventional in vitro fertilization. Andrology 2020;8:1107-1116.

20. Kirkegaard K, Kesmodel US, Hindkjær JJ, Ingerslev HJ. Time-lapse parameters as predictors of blastocyst development and pregnancy outcome in embryos from good prognosis patients: a prospective cohort study. Hum Reprod 2013;28:2643-2651.

21. Siristatidis C, Komitopoulou MA, Makris A, Sialakouma A, Botzaki M, Mastorakos G, Salamalekis G, Bettocchi S, Palmer GA. Morphokinetic parameters of early embryo development via time lapse monitoring and their effect on embryo selection and ICSI outcomes: a prospective cohort study. J Assist Reprod Genet 2015;32:563-570.

22. Chen L, Li D, Ni X, Zhu L, Zhang N, Fang J, Jiang W, Wang J. Effects of the normal sperm morphology rate on the clinical and neonatal outcomes of conventional IVF cycles. Andrologia 2020;52:e13568.

23. Rubio I, Galán A, Larreategui Z, Ayerdi F, Bellver J, Herrero J, Meseguer M. Clinical validation of embryo culture and selection by morphokinetic analysis: a randomized, controlled trial of the EmbryoScope. Fertil Steril 2014;102:1287-1294.e5.

24. Meseguer M, Rubio I, Cruz M, Basile N, Marcos J, Requena A. Embryo incubation and selection in a time-lapse monitoring system improves pregnancy outcome compared with a standard incubator: a retrospective cohort study. Fertil Steril 2012;98:1481-1489.e10. 